\title{
Teaching Fourier optics for engineers
}

Joseph Shamir

Joseph Shamir, "Teaching Fourier optics for engineers," Proc. SPIE 2525, 1995 International Conference on Education in Optics, (13 October 1995); doi: $10.1117 / 12.224005$

SPIE Event: SPIE's 1995 International Symposium on Optical Science, Engineering, and Instrumentation, 1995, San Diego, CA, United States 


\title{
Teaching Fourier optics for engineers
}

\author{
Joseph Shamir
}

\author{
Department of Electrical Engineering \\ Technion - Israel Institute of Technology, Haifa 32000, Israel
}

\begin{abstract}
A conventional optical system can be regarded as a special type of linear system and can thus be treated accordingly. The scalar representation in its paraxial approximation lends itself to a particularly simple analysis. Employing an operator representation from a linear-systems point of view, introduces the senior engineering student to Fourier optics in the most natural and straight forward way. This approach eliminates the need for manipulating complicated integral expressions and leads to better insight into the physical processes.
\end{abstract}

\section{INTRODUCTION}

The approach presented in this paper is implemented, for more than ten years, as part of a senior level course on electro-optical systems. The first part of this course is dedicated to an extensive treatment of Fourier optics [1] which is covered in about 18 class hours including lectures and exercises. This is less than half the time required by conventional texts, thanks to the shorthand notation of the Operator Algebra [2]-[5]. As a prerequisite for this course, it is assumed that the students have a basic background of linear-systems analysis and electromagnetic field theory.

The course starts with an introductory presentation of the solution to the homogeneous wave equation which is then successively approximated by quasi-monochromatic, scalar, and finally, paraxial approximations. The notion of wavefront is introduced with the specific examples of plane waves and spherical waves that can be used for the linear decomposition of more complex wave fronts.

The main theme of the course follows after these introductory remarks with a spherical wave, generated by a point source, considered as the impulse response of free space. After this, the Fresnel-Kirchhoff diffraction integral [1] is a straight forward consequence of the linearity of the wave equation. The operator notation provides various shorthand forms of the diffraction integral and generates an excellent tool to analyze, understand, and synthesize optical systems.

The rest of this paper is derived from the notes of this course and may serve as the framework for similar courses. The references should be helpful for filing the gaps, preparing examples and expanding the scope.

\section{ANALYTIC SIGNAL REPRESENTATION}

The propagation of light and its macroscopic interaction with material objects can, in most cases, be represented by Maxwell's equations. These equations are the fundamentals of classical electromagnetic theory, but they can be solved exactly only for highly simplified conditions. Fortunately, a wide variety of physical phenomena can be accounted for and described fairly well by employing several simplifying assumptions.

Maxwell's equations can be manipulated to derive the wave equation, the solution of which describes the propagation of electromagnetic fields, the electric field vector, $\mathbf{E}(x, y, z, t)$, and the magnetic field vector, $\mathbf{H}(x, y, z, t)$. Since Maxwell's equations contain a unique relationship between these two vectors, one of them is usually adequate for a complete representation of the field. For mathematical convenience the field vectors are evaluated as complex analytic signals with the understanding that only their real 
parts have actual physical meaning. Of course, some useful information about the behavior of the fields is contained in the imaginary part as well.

The orientation of the field vector determines the wave's polarization state. If the orientation is constant in space and time, then the wave is called plane polarized (or linearly polarized). If the orientation rotates the polarization is said to be circular or elliptic while a completely random variation results in unpolarized waves. Isotropic media have no preferred orientations, therefore they cannot be sensitive to the polarization state of light propagating through them.

In many practical situations only isotropic media are involved and the anisotropic character of optical components can be neglected. For these situations the scalar approximation, where the vector nature of the fields is ignored, can be readily applied.

Advanced laser technology provided a wide selection of narrow band-width light sources. The light emerging from an idealized source of this kind can be represented by a coherent, quasi-monochromatic signal of the form,

$$
\mathbf{E}(x, y, z, t) \rightarrow \mathbf{E}^{\prime}(x, y, z, t) e^{j \omega t}
$$

where the variation of the complex amplitude (or complex envelope), $\mathbf{E}^{\prime}(x, y, z, t)$, in time is assumed to be slow as compared to the mean frequency, $\nu=\omega / 2 \pi$.

\section{WAVE FRONTS}

In macroscopic optical systems light has usually a preferred direction of propagation. In such systems it is convenient to decompose the spatial function of the complex amplitude in a similar way as the temporal part in Eq. (1). Doing this within the framework of the scalar approximation, the complex amplitude can be written in the form,

$$
\mathbf{E}^{\prime}(x, y, z, t) \rightarrow u(x, y, z, t)=A(x, y, z, t) e^{-j[\mathrm{k}(x, y, z, t) \cdot \mathbf{r}]+\phi(x, y, z, t)}
$$

where all quantities on the right-hand side of the equation are real. The coordinate vector, $\mathbf{r}$, is given by,

$$
\mathbf{r}=x \hat{x}+y \hat{y}+z \hat{z} ; \quad|\mathbf{r}| \equiv r=\sqrt{x^{2}+y^{2}+z^{2}}
$$

with . denoting unit vectors. In an isotropic and homogeneous medium the orientation of the wave vector, $\mathbf{k}$, is in the direction of the Pointing vector, i.e. the direction of energy propagation, and its magnitude is related to the wavelength of the radiation, $\lambda$, by the relation, $|\mathrm{k}| \equiv k=2 \pi / \lambda$. Like in the temporal decomposition, Eq. (2) has a useful meaning only if the spatial variations of $A, \phi$ and $\mathrm{k}$ are slow as compared to the wavelength, $\lambda$. If we equate the scalar product of the wave vector and the radius vector, to a constant,

$$
\mathbf{k}(x, y, z, t) \cdot \mathbf{r}=x k_{x}(x, y, z, t)+y k_{y}(x, y, z, t)+z k_{z}(x, y, z, t)=\text { const }
$$

we obtain the equation of a surface in three-dimensional space. Ignoring the possible variation in time this surface is usually referred to as the wave front.

Maxwell's equations are linear in the electromagnetic field components as long as the characteristics of the media in the system do not depend on the fields themselves. Throughout this paper only linear media are considered, thus the principle of linear superposition holds. That is, a general solution can be found by a linear superposition of partial solutions. Stated in a different way, any complicated wave front can be expressed in terms of a linear superposition of simpler wave fronts, or elementary wave fronts. From a mathematical point of view this is similar to the decomposition of a complicated function into 
a set of orthonormal functions like a Fourier decomposition into a set of cosine and sine functions. Two such simple idealized wave fronts are useful in particularly: The planar and spherical wave fronts. For a planar wave front $\mathbf{k}$ is a constant and the relation,

$$
\mathbf{k} \cdot \mathbf{r}=x k_{x}+y k_{y}+z k_{z}=\text { const. }
$$

represents a plane in three-dimensional space. In particular, a plane wave propagating in the positive $z$ direction has the wave vector, $\mathrm{k}=k \hat{z}$ and Eq. (5) represents planes normal to the $z$-axis.

A spherical wave with its center at the origin is represented by the wave vector $\mathbf{k}=k \hat{r}$ thus

$$
\mathbf{k} \cdot \mathbf{r}=k r=\text { const. }
$$

is a sphere with radius $r=$ const. $/ k$. Both, the ideal spherical and plane waves are idealized mathematical entities and cannot be realized in practice since they involve singularities or infinite system requirements. Nevertheless, they are good approximations for various situations and any wave form can be represented as a linear superposition of an infinite number of either of these fundamental wave forms.

\section{DIFFRACTION}

Our final objective is to determine the complex amplitude distribution over some output region of an optical system when the distribution over an input region is known. In most practical cases the optical system is enclosed between two planes that represent the output and input regions (Fig. 1). Starting from propagation in free space, we shall show that the corresponding complex amplitude distributions are related to each other by a linear operator which we shall call a transfer operator. For this purpose we
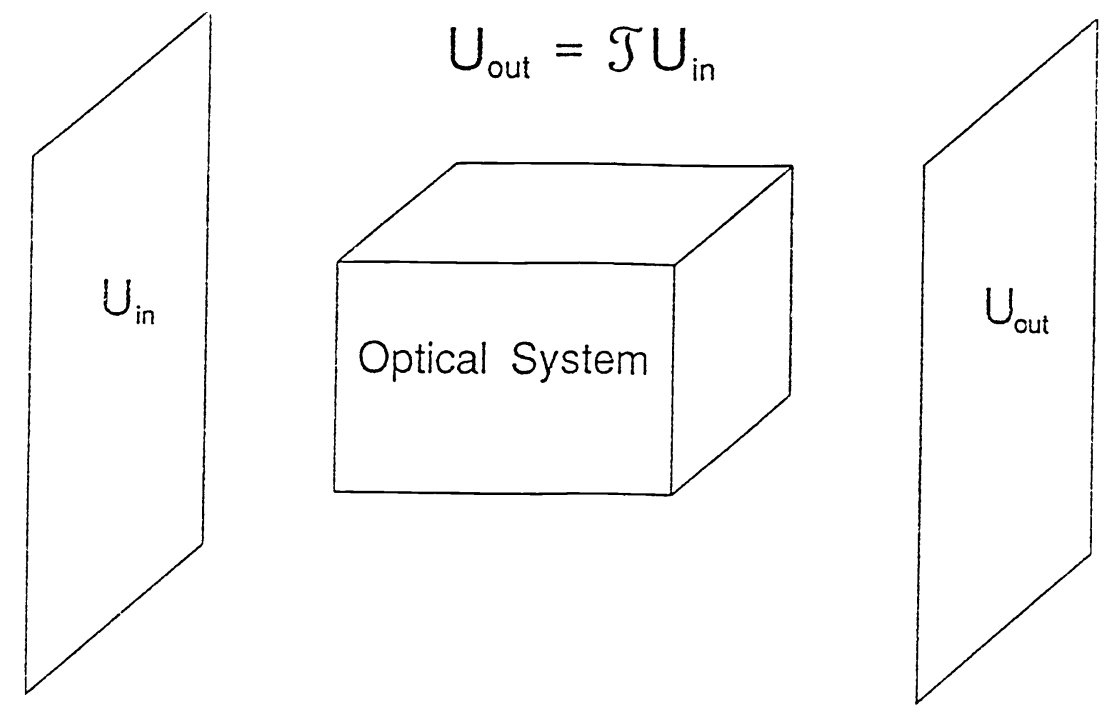

Figure 1: General optical system described by its transfer operator.

note that an ideal point source of unit magnitude, located at the origin, emits a spherical wave given by,

$$
\mathcal{O}(r)=\frac{1}{j \lambda r} e^{-j k r}
$$


Apart from the spherical wave front, this expression contains the $1 / r$ dependence which is required for energy conservation and the normalization factor, $j \lambda$, which will be justified later. A coherent radiating object can be represented as a distribution of point sources. Since Maxwell's equations are linear, the field distribution due to several sources will be a linear superposition of the field from all individual contributions. This is analogous to a linear system which responds to an input by a linear superposition of its components. Considering free space as a position invariant linear system, the spherical wave of Eq. (7) can be considered as its impulse response, or point spread function (PSF). Accordingly, if the source distribution is given by a function $u_{i n}(x, y, z, t)$, the complex amplitude distribution at a different location can be evaluated by the convolution integral,

$$
u_{\text {out }}(x, y, z, t)=u_{\text {in }}(x, y, z, t) * \mathcal{O}(r)
$$

where we assumed that the speed of light is infinite as compared to the size of the system and time delays can be ignored accordingly. With this assumption in mind, the time variable will be suppressed in the following discussions, for the sake of simplicity. The convolution is, in principle, to be evaluated in three dimensions. In most optical systems, however, the source distribution is given over some plane and the output is detected over another plane, parallel to the input plane and situated at some distance $d$ (see Fig. 1). It is convenient, therefore, to choose a coordinate system where light propagates mainly in the positive $z$ direction and the $x y$ plane coinciding with the input plane. The output plane is also normal to the $z$-axis at $z=d$.

Even for this relatively simple system, the convolution integral of Eq. (8) is quite complicated. This, however, can be substantially simplified if the source and output regions are small as compared to the distance $d$. In this case we may use the paraxial approximation which concerns the distance, $r$ (Eq. 3 ), which can be written in the form.

$$
r=z \sqrt{1+\frac{x^{2}+y^{2}}{z^{2}}} \approx z\left(1+\frac{x^{2}+y^{2}}{2 d^{2}}\right) .
$$

Using this paraxial approximation for the PSF of free space (7), it can be reduced to,

$$
\mathcal{O}(r)=\frac{e^{j k d}}{j \lambda d} \mathcal{Q}\left[\frac{1}{d}\right]
$$

where we introduced the quadratic phase factor,

$$
\mathcal{Q}\left[\frac{1}{d}\right] \equiv e^{-j \frac{k}{2 d}\left(x^{2}+y^{2}\right)}
$$

and approximated $r$ in the denominator by $d$. Using Eq. (10) for the PSF, the convolution integral (8) reduces to two dimensions and can be written in the form,

$$
u_{\text {out }}(x, y, d)=\frac{e^{j k d}}{j \lambda d} \mathcal{Q}\left[\frac{1}{d}\right] * u_{\text {in }}(x, y, 0)
$$

The above relation is a shorthand for the Fresnel-Kirchhoff diffraction integral as can be verified by writing explicitly the convolution operation:

$$
u_{\text {out }}(x, y, d)=\frac{e^{j k d}}{j \lambda d} \iint_{-\infty}^{\infty} e^{-j \frac{k}{2 d}\left[\left(x-x^{\prime}\right)^{2}+\left(y-y^{\prime}\right)^{2}\right]} u_{i n}\left(x^{\prime}, y^{\prime}, 0\right) d x^{\prime} d y^{\prime}
$$


Evaluating the squares in the exponent, rearranging terms and resubstituting the notation for the quadratic phase factor, this expression can be rewritten in the form,

$$
u_{\text {out }}(x, y, d)=\frac{e^{j k d}}{j \lambda d} \mathcal{Q}\left[\frac{1}{d}\right] \iint_{-\infty}^{\infty} e^{j \frac{k}{d}\left(x x^{\prime}+y y^{\prime}\right)} \mathcal{Q}\left[\frac{1}{d}\right] u_{i n}\left(x^{\prime}, y^{\prime}, 0\right) d x^{\prime} d y^{\prime} .
$$

In this expression the quadratic phase factors are considered as operators in the sense that their variables are to be taken the same as those of the expression on their right. Continuing this line of argument we observe that the integral is a properly scaled, two-dimensional Fourier transformation (FT). To simplify the notation we define a generic FT operator by the relation,

$$
\mathcal{F} f(x, y)=\iint_{-\infty}^{\infty} e^{j 2 \pi\left(x x^{\prime}+y y^{\prime}\right)} f\left(x^{\prime}, y^{\prime}\right) d x^{\prime} d y^{\prime}
$$

and a scaling operator,

$$
\mathcal{V}[a] f(x, y)=f(a x, a y)
$$

for any two-dimensional function $f(x, y)$. Each operator is assumed to operate on the whole expression on its right, unless enclosed in brackets. It should be noted that the definition of the FT operator is correct mathematically but it needs a proper scaling to make it dimensionally and physically meaningful.

With the help of the operators defined above, the diffraction integral can be written in the form,

$$
u_{\text {out }}(x, y, d)=\frac{e^{j k d}}{j \lambda d} \mathcal{Q}\left[\frac{1}{d}\right] \mathcal{V}\left[\frac{1}{\lambda d}\right] \mathcal{F} \mathcal{Q}\left[\frac{1}{d}\right] u_{i n}(x, y, 0)
$$

or,

$$
u_{\text {out }}(x, y, d)=\mathcal{R}[d] u_{\text {in }}(x, y, 0)
$$

where we defined the free space propagation operator (FPO) by the relation,

$$
\mathcal{R}[d]=\frac{e^{j k d}}{j \lambda d} \mathcal{Q}\left[\frac{1}{d}\right] \mathcal{V}\left[\frac{1}{\lambda d}\right] \mathcal{F} \mathcal{Q}\left[\frac{1}{d}\right] .
$$

Two other expressions for the FPO can be derived directly from Eq. (12): First of all, one may directly write,

$$
\mathcal{R}[d]=\frac{e^{j k d}}{j \lambda d} \mathcal{Q}\left[\frac{1}{d}\right] * .
$$

Alternatively, we may operate on the whole expression by the FT operator,

$$
\mathcal{F} u_{\text {out }}(x, y, d)=\frac{e^{j k d}}{j \lambda d}\left\{\mathcal{F} \mathcal{Q}\left[\frac{1}{d}\right]\right\} \mathcal{F} u_{\text {in }}(x, y, 0)
$$

where the convolution theorem [1] of the FT was used. From conventional Fourier analysis we obtain that the FT of a quadratic phase factor is a quadratic phase factor too:

$$
\mathcal{F} \mathcal{Q}\left[\frac{1}{d}\right]=j \lambda d \mathcal{Q}\left[-\lambda^{2} d\right]
$$

Substituting into Eq. (21) and performing an inverse FT on the result, leads to,

$$
u_{\text {out }}(x, y, d)=e^{j k d} \mathcal{F}^{-1} \mathcal{Q}\left[-\lambda^{2} d\right] \mathcal{F} u_{\text {in }}(x, y, 0) \text {. }
$$


Thus the third expression for the FPO can be written as,

$$
\mathcal{R}[d]=e^{j k d} \mathcal{F}^{-1} \mathcal{Q}\left[-\lambda^{2} d\right] \mathcal{F} .
$$

Relation (22) which was used to derive this last representation of the FPO is one of the commutation relations among the three basic operators. It is useful to list some of the others here too: By their definition, the $\mathcal{Q}$ and $\mathcal{V}$ operators satisfy the commutation relations,

$$
\begin{gathered}
\mathcal{Q}[a] \mathcal{Q}[b]=\mathcal{Q}[a+b] \\
\mathcal{V}[a] \mathcal{V}[b]=\mathcal{V}[a b] \\
\mathcal{V}[a] \mathcal{Q}[b]=\mathcal{Q}\left[a^{2} b\right] \mathcal{V}[a]
\end{gathered}
$$

Other relations can be readily derived from Fourier analysis:

$$
\begin{gathered}
\mathcal{V}[a] \mathcal{F}=\frac{1}{|a|^{2}} \mathcal{F} \mathcal{V}\left[\frac{1}{a}\right] ; \\
\mathcal{F F}=\mathcal{V}[-1] ; \quad \mathcal{F F}^{-1}=1 ;
\end{gathered}
$$

At this point we are equipped with the basic tools of diffraction theory and can also justify the normalization factor used in the definition of the spherical wave of Eq. (7). This factor was chosen in such a way that a plane wave propagating in the $z$ direction should retain its form. Since the complex amplitude distribution of such a plane wave is constant, this constant must be retained, except for the longitudinal phase accumulated during propagation. Operating with the FPO of Eq. (19) on a constant, $c$, we obtain the output distribution as,

$$
u_{\text {out }}=\mathcal{R}[d] c=\frac{e^{j k d}}{j \lambda d} \mathcal{Q}\left[\frac{1}{d}\right] \mathcal{V}\left[\frac{1}{\lambda d}\right] \mathcal{F} \mathcal{Q}\left[\frac{1}{d}\right] c
$$

Using relation (22) we obtain,

$$
u_{\text {out }}=\mathcal{R}[d] c=e^{j k d} \mathcal{Q}\left[\frac{1}{d}\right] \mathcal{V}\left[\frac{1}{\lambda d}\right] \mathcal{Q}\left[-\lambda^{2} d\right] c
$$

Substitution of the commutation relations Eqs. (27) and (25) reduces this relation to,

$$
u_{\text {out }}=\mathcal{R}[d] c=e^{j k d} c,
$$

as it should be, proving the proper choice of the normalization constant.

To end this section we point out that the region of validity of the approximations underlying the above treatment constitute the Fresnel region while the Fraunhofer region, the far field, is obtained for large distances where $\mathcal{Q}[1 / d] \rightarrow 1$ and the FPO (Eq. 19) reduces to a FT.

\section{THIN OPTICAL ELEMENTS}

In the previous section we described the propagation of a complex amplitude distribution through free space. To make an optical system one needs to assemble a set of optical components and place them at well defined positions. This section is devoted to the definition of the most fundamental optical elements, the thin dielectric slab, the thin prism and the thin lens. A thin optical element is defined as an element which operates on the complex amplitude as a multiplicative factor and diffraction can be ignored within 

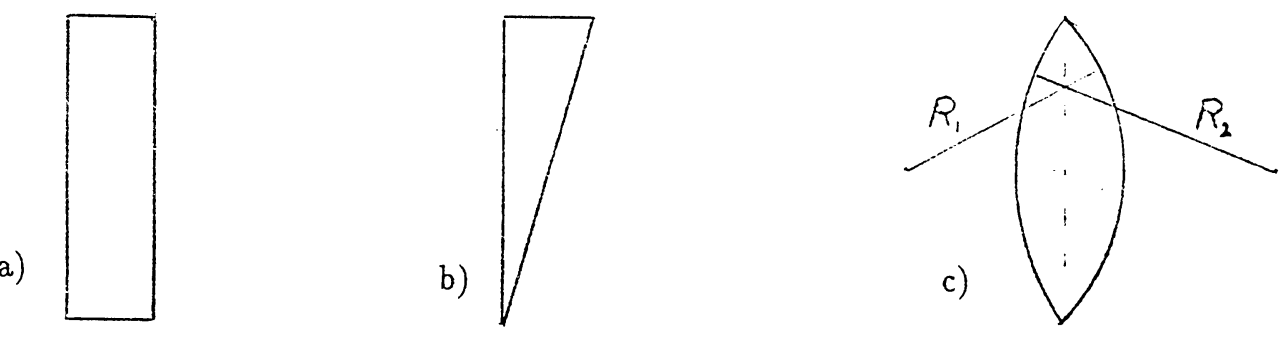

Figure 2: Thin optical elements. a) the dielectric slab; b) the thin prism; c) the thin lens

the element. In other words, a thin optical element can be compressed into an equivalent "transparency" of zero thickness. A distribution $u_{i n}$, incident on a thin optical element will emerge as,

$$
u_{\text {out }}=T u_{\text {in }}
$$

where $T$ is the transfer function of the element.

\subsection{The thin dielectric slab}

The thin dielectric slab (Fig. 2a) adds a constant phase to a propagating wave (ignoring Fresnel reflections). A plane wave, $\exp (-j k z)$, incident on a dielectric slab of thickness $d$ and refractive index $n$, will emerge as $\exp [-j k(z+n d)]$ instead of $\exp [-j k(z+d)]$, without the slab. Thus, the effect of the slab may be described by the transfer function $T$ according to the relations,

$$
e^{-j k(z+n d)}=T e^{-j k(z+d)} \rightarrow T=e^{-j k d(n-1)}
$$

\subsection{The thin prism}

The thin prism (Fig. $2 \mathrm{~b}$ ) is a dielectric slab with its thickness depending on position. In the special case shown, $d=a x$, where $a$ is $\tan (\alpha)$ ( $\approx \alpha$ for the small angle of a thin prism). Substituting into Eq. (34) we obtain,

$$
T=e^{-j k d(x)(n-1)}=e^{-j k a x(n-1)} \equiv \mathcal{G}[a(n-1)]
$$

where we defined the linear phase factor, $\mathcal{G}[\vec{a}]$ which, in general, has a vector label since the prism can be oriented in any direction. In fact, the linear phase represents a plane wave propagating in an off axis direction when the paraxial approximation is used. Since the linear phase factor is frequently encountered, it is useful to list some of its relations with other operators:

$$
\begin{gathered}
\mathcal{G}[\vec{a}] \mathcal{G}[\vec{b}]=\mathcal{G}[\vec{a}+\vec{b}] \\
\mathcal{V}[b] \mathcal{G}[\vec{m}]=\mathcal{G}[\vec{m} b] \mathcal{V}[b] \\
\mathcal{F} \mathcal{G}[\vec{s}]=\mathcal{S}[\vec{s} / \lambda] \mathcal{F}
\end{gathered}
$$


where $\mathcal{S}\left[\hat{(x)} s_{x}+\hat{y} s_{y}\right]$ is the shift operator defined by,

$$
\mathcal{S}\left[\hat{(x)} s_{x}+\hat{y} s_{y}\right] f(x, y)=f\left(x-s_{x}, y-s_{y}\right) .
$$

It should be noted here that relation (38) is to be considered always in conjunction with a scaling operator to make it dimensionally correct.

\subsection{The thin lens}

The thin lens (Fig. 2c) is a dielectric medium enclosed between two spherical surfaces. The position dependent thickness can be determined from simple geometrical considerations. To a first approximation we obtain $d=a-\left(1 / 2 R_{1}+1 / 2 R_{2}\right)\left(x^{2}+y^{2}\right)$, where $R_{1}$ and $R_{2}$ are the two radii of curvature and $a$ is the thickness of the lens at its center. Thus the transfer function of the thin lens can be written as,

$$
T=e^{-j k d(x)(n-1)}=e^{-j k a(n-1)} \mathcal{Q}\left[-\frac{1}{f}\right]
$$

where the focal length, $f$ is defined by,

$$
\frac{1}{f}=(n-1)\left(\frac{1}{R_{1}}+\frac{1}{R_{2}}\right)
$$

In the rest of this work the constant phase factor in Eq. (40) will be ignored and the lens transfer function will be represented by the quadratic phase factor.

\section{OPTICAL SYSTEMS CONTAINING THIN LENSES}

The simplest operative optical system is composed of a thin lens enclosed between two free space sections (Fig. 3). The input distribution is operated on by an FPO through a distance $a$, the result is

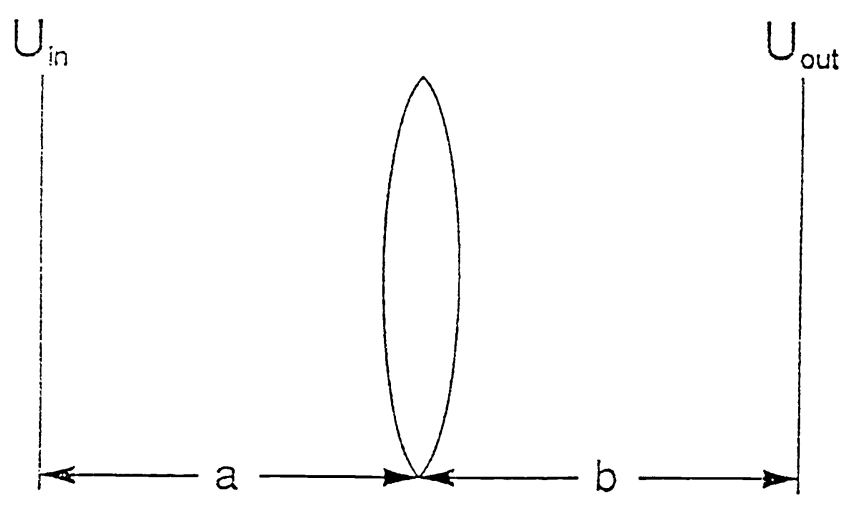

Figure 3: Optical system with a thin lens of focal length $f$

multiplied by the quadratic phase factor of the lens and then a second FPO operates through a distance $b$. Thus the whole optical system can be represented by a transfer operator, $\mathcal{T}$, given by,

$$
\mathcal{T}=\mathcal{R}[b] \mathcal{Q}\left[-\frac{1}{f}\right] \mathcal{R}[a] .
$$


This expression can be manipulated using the relations among the operators to derive various functions of the optical system. Two examples will be presented here.

\subsection{Imaging with a thin lens}

Substituting the representation of Eq. (19) for the two FPOs in Eq. (42) we obtain,

$$
\mathcal{T}=\frac{e^{j k(b+a)}}{-\lambda^{2} b a} \mathcal{Q}\left[\frac{1}{b}\right] \mathcal{V}\left[\frac{1}{\lambda b}\right] \mathcal{F} \mathcal{Q}\left[\frac{1}{b}\right] \mathcal{Q}\left[-\frac{1}{f}\right] \mathcal{Q}\left[\frac{1}{a}\right] \mathcal{V}\left[\frac{1}{\lambda a}\right] \mathcal{F} \mathcal{Q}\left[\frac{1}{a}\right]
$$

where the constant factors from the two FPOs were combined. Using relation (25) leads to,

$$
\mathcal{T}=\frac{e^{j k(b+a)}}{-\lambda^{2} b a} \mathcal{Q}\left[\frac{1}{b}\right] \mathcal{V}\left[\frac{1}{\lambda b}\right] \mathcal{F} \mathcal{Q}\left[\frac{1}{b}-\frac{1}{f}+\frac{1}{a}\right] \mathcal{V}\left[\frac{1}{\lambda a}\right] \mathcal{F} \mathcal{Q}\left[\frac{1}{a}\right]
$$

Inserting the classical imaging condition,

$$
\frac{1}{b}+\frac{1}{a}=\frac{1}{f}
$$

eliminates the central $\mathcal{Q}$ factor $(\mathcal{Q}[0]=1)$. Using subsequently the various commutation relations, (Eqs. $29,28,27,26$ and 25$)$ reduces the transfer operator to,

$$
\mathcal{T}=\frac{a}{b} e^{j k(b+a)} \mathcal{Q}\left[\frac{1}{b}\left(1+\frac{a}{b}\right)\right] \mathcal{V}\left[-\frac{a}{b}\right] .
$$

The meaning of this operator is that the input distribution is reconstructed with a modified scale (magnification is $-b / a$ with the proper intensity adjustment by the constant factor) and a quadratic phase distortion which can be ignored if only the intensity is measured. To eliminate the quadratic phase, an additional lens is required. For example, a field lens, having a focal length $f^{\prime}$ given by the relation,

$$
\frac{1}{f^{\prime}}=\frac{1}{b}\left(1+\frac{a}{b}\right)
$$

can be attached to the output plane.

\subsection{Fourier transformation with a thin lens}

Returning to the generic transfer operator of Eq. (42) we may write the left-hand side FPO in the form of Eq. (19) and the right-hand side FPO in the form of Eq. (24) to obtain,

$$
\mathcal{T}=\frac{e^{j k(b+a)}}{j \lambda b} \mathcal{Q}\left[\frac{1}{b}\right] \mathcal{V}\left[\frac{1}{\lambda b}\right] \mathcal{F} \mathcal{Q}\left[\frac{1}{b}\right] \mathcal{Q}\left[-\frac{1}{f}\right] \mathcal{F}^{-1} \mathcal{Q}\left[-\lambda^{2} a\right] \mathcal{F}
$$

If we take $b=f$, the middle $\mathcal{Q}$ operators are canceled and then the product $\mathcal{F F}^{-1}$ is canceled too. Commuting now $\mathcal{V}$ with the $\mathcal{Q}$ on its right leads to,

$$
\mathcal{T}=\frac{e^{j k(f+a)}}{j \lambda f} \mathcal{Q}\left[\frac{1}{f}\left(1-\frac{a}{f}\right)\right] \mathcal{V}\left[\frac{1}{\lambda f}\right] \mathcal{F}
$$

This is a FT with the proper scale and a quadratic phase factor which can be eliminated by taking $a=b=f$.

In this section we treated the generic optical system with one lens. This system can be considered as a building block which can be cascaded to construct more complicated optical system. For example, a simple telescope and an optical correlator are composed of a cascade of two FT systems, while a microscope is a cascade of two imaging systems. 


\section{CONCLUSIONS}

We presented here the fundamentals of Fourier optics from a linear-systems point of view. This framework is adequate for solving most problems of optical system design and analysis encountered in the course and can be extended to such fields as holography [4, 5], speckle pattern analysis [6], and aberrations [7]. The treatment can be extended further and made mathematically more rigorous by involving canonical operators and group theory [8]-[14]. In the more advanced and rigorous representation the operators are defined in a normalized form where also the FT operator is dimensionally correct. However, there the operator manipulations are slightly more complicated.

\section{References}

[1] see, for example, J. W. Goodman. Introduction to Fourier Optics, McGraw-Hill Book Co., San Francisco 1968.

[2] M. Nazarathy and J. Shamir, "Fourier optics described by operator algebra" J. Opt. Soc. Am. Vol. 70, pp. 150-158, (1980).

[3] J. Shamir, "Cylindrical Lens Systems Described by Operator Algebra", Appl. Opt. Vol. 18, pp. 4195-4202, (1979).

[4] M. Nazarathy and J. Shamir, "Holography described by operator algebra" J. Opt. Soc. Am. Vol. 71, pp. 529-541, (1981).

[5] M. Nazarathy and J. Shamir, "Wavelength Variation in Fourier Optics and Holography Described by Operator Algebra", Israel J. Tech. Vol. 18, pp. 224-23l, (1980).

[6] Y. Fainman, J. Shamir and E. Lenz, "Static and Dynamic Behavior of Speckle Patterns Described by Operator Algebra", Appl. Opt. Vol. 20, pp. 3526-3538 (1981).

[7] J. Shamir and R.A. Chipman, "Polarization aberration effects propagated in optical systems" J. Mod. Opt. Vol. 38, pp. 327-347, (1991)

[8] M. Nazarathy and J. Shamir, "First-Order Optics - A Canonical Operator Representation - Lossless Systems", J. Opt. Soc. Am. Vol. 72, pp. 356-364 (1982).

[9] M. Nazarathy and J. Shamir, "First-Order Optics - Operator Representation for Systems with Loss or Gain", J. Opt. Soc. Am. Vol. 72, pp. 1398-1408 (1982).

[10] M. Nazarathy, A. Hardy and J. Shamir, "Generalized Mode Propagation in First-Order Optical Systems with Loss or Gain", J. Opt. Soc. Am. Vol. 72, pp. 1409-1420 (1982).

[11] M. Nazarathy, A. Hardy and J. Shamir, "Generalized Mode Theory of Conventional and PhaseConjugate Resonators", J. Opt. Soc. Am., Vol. 73, pp. 576-586 (1983).

[12] M. Nazarathy, J. Shamir and A. Hardy, "Nonideal Phase- Conjugate Resonators - A Canonical Operator Analysis", J. Opt. Soc. Am., Vol. 73, pp. 587-593 (1983).

[13] M. Nazarathy and J. Shamir, "Phase Conjugacy and Symmetries in General Optical Systems", J. Opt. Soc. Am., Vol. 73, pp. 910-915 (1983).

[14] M. Nazarathy, A. Hardy and J. Shamir, "Misaligned First- Order Optics: Canonical Operator Theory" J. Opt. Soc. Am. Vol. A3, pp. 1360-1369, (1986) 